\title{
Algebraic structures of multipartite quantum systems
}

\author{
Hoshang Heydari \\ Institute of Quantum Science, Nihon University, \\ 1-8 Kanda-Surugadai, Chiyoda-ku, Tokyo 101-8308, Japan
}

\begin{abstract}
We investigate the relation between multilinear mappings and multipartite states. We show that the isomorphism between multilinear mapping and tensor product completely characterizes decomposable multipartite states in a mathematically well-defined manner.
\end{abstract}

\section{Introduction}

The characterization of multipartite state is a fascinating subject in the field of fundamental quantum theory with interesting applications in quantum information and quantum computing. There are many multipartite states which can be used for different algorithm or scheme for quantum computation. For example entangled cluster states are the building block for one-way quantum computer as scheme for universal quantum computation. In recent years, we have witnessed some progress in quantification and classification of multipartite states, but this problem is still open and needs further investigation.

In this paper we will discuss the structure of multipartite product state in a clear and abstract algebraic way. Our main interest are multilinear mapping or $m$-linear mapping of complex vector spaces. We will establish an isomorphism between this maps and tensor product states. In section 2 we will give an introduction to the structure of bilinear mapping and condition for which we have isomorphic mapping between this bilinear mapping and tensor product. We will also show that this construction defines the separable set of general bipartite states. Moreover, we will establish a relation between this construction and the concurrence. In section [3, we will generalize our result from bilinear to multilinear or $m$-linear mapping and the construction of tensor product for such mapping. We will show that this construction describes the separable set of general multipartite states. Finally, we will establish a relation between this algebraic construction and the generalized concurrence. An introduction to theory of multilinear mapping and algebra can be found in 1 which is also our main reference. 


\section{Bilinear mapping and bipartite state}

In this section we will give an introduction to bilinear mapping and tensor product. Let $\mathrm{V}_{1}, \mathrm{~V}_{2}$ be two linear spaces and consider the mapping

$$
\Phi: \mathcal{V}_{1} \times \mathcal{V}_{2} \longrightarrow \mathcal{M}
$$

This map is called bilinear if it satisfies the following condistions

1. $\left.\Phi\left(\lambda\left|\psi_{1}^{1}\right\rangle+\mu\left|\psi_{1}^{2}\right\rangle,\left|\psi_{2}\right\rangle\right)=\lambda \Phi\left(\left|\psi_{1}^{1}\right\rangle,\left|\psi_{2}\right\rangle\right)\right)+\mu \Phi\left(\left|\psi_{1}^{2}\right\rangle,\left|\psi_{2}\right\rangle\right)$ for all $\left|\psi_{1}^{1}\right\rangle,\left|\psi_{1}^{2}\right\rangle \in$ $\mathcal{V}_{1},\left|\psi_{2}\right\rangle \in \mathcal{V}_{2}$, and $\lambda, \mu \in \mathcal{N}$

2. $\left.\Phi\left(\left|\psi_{1}\right\rangle, \lambda\left|\psi_{2}^{1}\right\rangle+\mu\left|\psi_{2}^{2}\right\rangle\right)=\lambda \Phi\left(\left|\psi_{1}\right\rangle,\left|\psi_{2}^{1}\right\rangle\right)\right)+\mu \Phi\left(\left|\psi_{1}\right\rangle,\left|\psi_{2}^{2}\right\rangle\right)$ for all $\left|\psi_{2}^{1}\right\rangle,\left|\psi_{2}^{2}\right\rangle \in$ $\mathcal{V}_{2},\left|\psi_{1}\right\rangle \in \mathcal{V}_{1}$

for a linear space $\mathcal{N}$. We call this bilinear algebra a bilinear function if the linear space $\mathcal{N}=\mathcal{M}$. In this paper, we are mostly interested in complex vector spaces and specially finite dimensional. The most important observation about bilinear mapping is that the set of all vectors $\mathcal{S}$ in $\mathcal{M}$ of the form $\Phi\left(\left|\psi_{1}\right\rangle,\left|\psi_{2}\right\rangle\right)$ for $\left|\psi_{1}\right\rangle \in \mathcal{V}_{1}$ and $\left|\psi_{2}\right\rangle \in \mathcal{V}_{2}$ is not in general a linear subspace of the target space $\mathcal{M}$. To make this important point clear we will give an example based on pair qubits. In this case we have $\mathcal{V}_{1}=\mathcal{V}_{2}=\mathbb{C}^{2}$ and $\mathcal{M}=\mathbb{C}^{4}$. Let also $\left|\psi_{1}\right\rangle=\alpha_{1}^{1}\left|e_{1}\right\rangle+\alpha_{2}^{1}\left|e_{1}\right\rangle$ and $\left|\psi_{2}\right\rangle=\alpha_{1}^{2}\left|e_{1}\right\rangle+\alpha_{2}^{2}\left|e_{1}\right\rangle$ In this case the bilinear mapping $\Phi\left(\left|\psi_{1}\right\rangle,\left|\psi_{2}\right\rangle\right)$ is defined by

$$
\Phi\left(\left|\psi_{1}\right\rangle,\left|\psi_{2}\right\rangle\right)=\alpha_{1}^{1} \alpha_{1}^{2}\left|f_{1}\right\rangle+\alpha_{1}^{1} \alpha_{2}^{2}\left|f_{2}\right\rangle+\alpha_{2}^{1} \alpha_{1}^{2}\left|f_{3}\right\rangle+\alpha_{2}^{1} \alpha_{2}^{2}\left|f_{4}\right\rangle
$$

where $\left|f_{j}\right\rangle$ is a basis for $\mathcal{M}$. Then a vector $|\psi\rangle=\sum_{j} \alpha^{j}\left|f_{j}\right\rangle \in \mathcal{M}$ is contained in the set $\mathcal{S}$ if and only if the components satisfy the following very important condition:

$$
\alpha^{1} \alpha^{4}=\alpha^{2} \alpha^{3} \text {. }
$$

This condition is exactly the separability condition for pair of qubits. Let us consider the a pure two qubit state $|\Psi\rangle=\sum_{i_{1}=0}^{1} \sum_{i_{2}=0}^{1} \alpha_{i_{1} i_{2}}\left|i_{1}\right\rangle \otimes\left|i_{2}\right\rangle$. Then for this state the separability condition is given by $\alpha_{00} \alpha_{11}=\alpha_{01} \alpha_{10}$. This equation also gives a well-known measure of entanglement called the concurrence $C(|\Psi\rangle)=2\left|\alpha_{00} \alpha_{11}-\alpha_{01} \alpha_{10}\right|$ for a pair of qubit [2].

Thus this is very important to investigate the algebra of product states for bipartite and multipartite states. In following sections we will use the notation $\operatorname{Im} \Phi$ for the subspace of $\mathcal{M}$ which is generated by the set $\mathcal{S}$.

Definition 2.0.1 Let $\mathrm{V}_{1}, \mathrm{~V}_{2}$ be two complex vector spaces and consider the mapping $\Phi: \mathcal{V}_{1} \times \mathcal{V}_{2} \longrightarrow \mathcal{M}$. Then the pair $(\Phi, \mathcal{M})$ is called a tensor product if and only if the following important conditions are satisfied:

- I: the image of bilinear mapping is equal the target space $\operatorname{Im} \Phi=\mathcal{M}$

- II: If there is bilinear mapping $\Psi: \mathcal{V}_{1} \times \mathcal{V}_{2} \longrightarrow \mathcal{N}$, where $\mathcal{N}$ is a arbitrary complex vector space, then there exists a linear mapping $\Theta \mathcal{M} \longrightarrow \mathcal{N}$ such that the following diagram

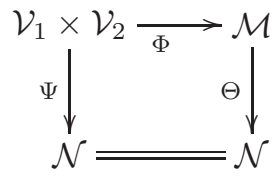

is commutative, that is $\Psi=\Theta \circ \Phi$. 
If $(\Phi, \mathcal{M})$ is a tensor product, then we denote $\mathcal{M}=\mathcal{V}_{1} \otimes \mathcal{V}_{2}$ and $\Phi\left(\left|\phi_{1}\right\rangle,\left|\phi_{2}\right\rangle\right)=$ $\left|\phi_{1}\right\rangle \otimes\left|\phi_{2}\right\rangle$. Moreover, the bilinearity is give by

1. $\left(\lambda\left|\psi_{1}^{1}\right\rangle+\mu\left|\psi_{1}^{2}\right\rangle\right) \otimes\left|\psi_{2}\right\rangle=\lambda\left|\psi_{1}^{1}\right\rangle \otimes\left|\psi_{2}\right\rangle+\mu\left|\psi_{1}^{2}\right\rangle \otimes\left|\psi_{2}\right\rangle$ for all $\left|\psi_{1}^{1}\right\rangle,\left|\psi_{1}^{2}\right\rangle \in \mathcal{V}_{1}$, $\left|\psi_{2}\right\rangle \in \mathcal{V}_{2}$, and $\lambda, \mu \in \mathcal{N}$

2. $\left.\left.\left|\psi_{1}\right\rangle \otimes\left(\lambda\left|\psi_{2}^{1}\right\rangle+\mu\left|\psi_{2}^{2}\right\rangle\right)=\lambda\left|\psi_{1}\right\rangle \otimes\left|\psi_{2}^{1}\right\rangle\right)\right)+\mu\left|\psi_{1}\right\rangle \otimes\left|\psi_{2}^{2}\right\rangle$ for all $\left|\psi_{2}^{1}\right\rangle,\left|\psi_{2}^{2}\right\rangle \in \mathcal{V}_{2}$, $\left|\psi_{1}\right\rangle \in \mathcal{V}_{1}$.

Next, we will give some elementary properties of the tensor product. For example every vector $0 \neq|\psi\rangle \in \mathcal{V}_{1} \otimes \mathcal{V}_{2}$ can be written as $|\psi\rangle=\sum_{i=1}^{k}\left|\psi_{1}^{i}\right\rangle \otimes\left|\psi_{2}^{i}\right\rangle$ for linearly independent vectors $\left|\psi_{1}^{i}\right\rangle$ and $\left|\psi_{2}^{i}\right\rangle$. To see that let us choose a representation of $|\psi\rangle$ such that it minimize the $k$. For $k=1$, it follows easily that $\left|\psi_{1}^{1}\right\rangle \neq 0$ and $\left|\psi_{2}^{1}\right\rangle \neq 0$ and so $\left|\psi_{1}^{1}\right\rangle$ and $\left|\psi_{2}^{1}\right\rangle$ are linearly independent vectors. Next, we show that the case for $k \geq 2$ is also correct. For a linearly dependent vector we can assume that $\left|\psi_{1}^{i}\right\rangle=\sum_{i=1}^{k-1} \gamma^{i}\left|\psi_{1}^{i}\right\rangle$, so we have

$$
\begin{aligned}
|\psi\rangle & =\sum_{i=1}^{k-1}\left|\psi_{1}^{i}\right\rangle \otimes\left|\psi_{2}^{i}\right\rangle+\sum_{i=1}^{k-1} \gamma^{i}\left|\psi_{1}^{i}\right\rangle \otimes\left|\psi_{2}^{k}\right\rangle \\
& =\sum_{i=1}^{k-1}\left|\psi_{1}^{i}\right\rangle \otimes\left(\left|\psi_{2}^{i}\right\rangle+\gamma^{i}\left|\psi_{2}^{k}\right\rangle=\sum_{i=1}^{k-1}\left|\psi_{1}^{i}\right\rangle \otimes\left|\psi_{2}^{i^{\prime}}\right\rangle .\right.
\end{aligned}
$$

This show that $k$ is not minimal. We can also show that $\left|\psi_{2}^{i}\right\rangle$ are linearly independent in the same way as above.

Now, let Let $\mathcal{V}_{1}, \mathcal{V}_{2}$ be two complex vector spaces and $\mathcal{V}_{1} \otimes \mathcal{V}_{2}$ be a tensor product for these spaces. Moreover, let $\mathcal{L}\left(\mathcal{V}_{1} \otimes \mathcal{V}_{2} ; \mathcal{M}\right)$ denotes linear mapping $\mathcal{V}_{1} \otimes \mathcal{V}_{2} \longrightarrow \mathcal{M}$ and $\mathcal{B}\left(\mathcal{V}_{1}, \mathcal{V}_{2} ; \mathcal{M}\right)$ denotes bilinear mapping $\mathcal{V}_{1} \times \mathcal{V}_{2} \longrightarrow \mathcal{M}$. Then, we have following isomorphism

$$
\mathcal{L}\left(\mathcal{V}_{1} \otimes \mathcal{V}_{2} ; \mathcal{M}\right) \longrightarrow \mathcal{B}\left(\mathcal{V}_{1}, \mathcal{V}_{2} ; \mathcal{M}\right)
$$

which is defined by $\Phi(\Theta)=\Theta \circ \otimes$ for all $\Theta \in \mathcal{L}\left(\mathcal{V}_{1} \otimes \mathcal{V}_{2} ; \mathcal{M}\right)$. The proof follows from conditions I and II for tensor product. Moreover, the correspondence between the linear map $\Psi \in \mathcal{L}\left(\mathcal{V}_{1} \otimes \mathcal{V}_{2} ; \mathcal{M}\right)$ and $\Theta \in \mathcal{B}\left(\mathcal{V}_{1}, \mathcal{V}_{2} ; \mathcal{M}\right)$ is visualize in following commutative diagram

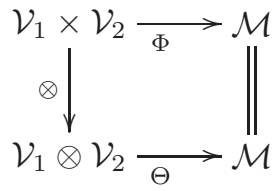

Thus we have the following proposition

Proposition 2.0.2 Let $\Psi \in \mathcal{L}\left(\mathcal{V}_{1} \otimes \mathcal{V}_{2} ; \mathcal{M}\right)$ be a bilinear map and $\Theta \in \mathcal{B}\left(\mathcal{V}_{1}, \mathcal{V}_{2} ; \mathcal{M}\right)$ be induced linear map. Then $\Theta$ is surjective and injective if and only if $\Phi$ satisfies the condition I and II of tensor respectively.

The map $\Theta$ is surjective follows from $\operatorname{Im} \Psi=\operatorname{Im} \Theta$. Now, if we assume that $\Theta$ is injective, then $(\operatorname{Im} \Psi, \Psi)$ is a tensor product for $\mathcal{V}_{1}$ and $\mathcal{V}_{2}$ and every bilinear mapping $\mathcal{V}_{1} \times \mathcal{V}_{2} \longrightarrow \mathcal{N}$ induces a linear mapping $\Upsilon: \operatorname{Im} \Psi \longrightarrow \mathcal{N}$ such that $\Phi\left(\left|\psi_{1}\right\rangle,\left|\psi_{2}\right\rangle\right)=\Upsilon \Psi\left(\left|\psi_{1}\right\rangle,\left|\psi_{2}\right\rangle\right)$. Next, $\Psi$ satisfies the condition II since if $\Theta$ is 
an extension of $\Upsilon$ to a map $\Theta: \mathcal{M} \longrightarrow \mathcal{N}$, then $\Phi\left(\left|\psi_{1}\right\rangle,\left|\psi_{2}\right\rangle\right)=\Theta \Psi\left(\left|\psi_{1}\right\rangle,\left|\psi_{2}\right\rangle\right)$. The converse follows by assuming that $\Psi$ satisfies the condition II and show that $\Theta$ is injective.

As an example let us look at the general bipartite states. For such system we have a bilinear mapping $\Sigma: \mathbb{C}^{N_{1}} \times \mathbb{C}^{N_{2}} \longrightarrow \mathrm{M}^{N_{1} \times N_{2}}$ defined by

$\left(\alpha_{1}^{1}, \alpha_{1}^{2}, \ldots, \alpha_{1}^{N_{1}}\right) \times\left(\alpha_{2}^{1}, \alpha_{2}^{2}, \ldots, \alpha_{2}^{N_{2}}\right) \longrightarrow\left(\begin{array}{cccc}\alpha_{1}^{1} \alpha_{2}^{1} & \alpha_{1}^{1} \alpha_{2}^{2} & \cdots & \alpha_{1}^{1} \alpha_{2}^{N_{2}} \\ \alpha_{1}^{2} \alpha_{2}^{1} & \alpha_{1}^{2} \alpha_{2}^{2} & \cdots & \alpha_{1}^{2} \alpha_{2}^{N_{2}} \\ \vdots & \vdots & \ddots & \vdots \\ \alpha_{1}^{N_{1}} \alpha_{2}^{1} & \alpha_{1}^{N_{1}} \alpha_{2}^{2} & \cdots & \alpha_{1}^{N_{1}} \alpha_{2}^{N_{2}}\end{array}\right)$

But for this bilinear mapping the pair $\left(\mathrm{M}^{N_{1} \times N_{2}}, \Sigma\right)$ is a tensor product of $\mathbb{C}^{N_{1}}$ and $\mathbb{C}^{N_{2}}$, that is

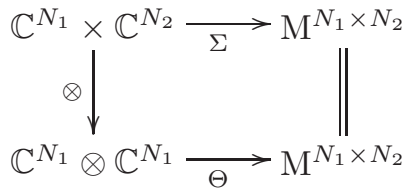

and thus represent the product states of general bipartite states. Let us consider the a general pure bipartite state $|\Psi\rangle=\sum_{i_{1}=0}^{N_{1}-1} \sum_{i_{2}=0}^{N_{2}-1} \alpha_{i_{1} i_{2}}\left|i_{1}\right\rangle \otimes\left|i_{2}\right\rangle$ and $\mathrm{M}^{N_{1} \times N_{2}}=\left\{\alpha_{i_{1} i_{2}} \in \mathbb{C}^{N_{1}} \times \mathbb{C}^{N_{2}}: \alpha_{k_{1} k_{2}} \alpha_{l_{1} l_{2}}=\alpha_{l_{1} k_{2}} \alpha_{k_{1} l_{2}}, \forall i_{j}=k_{j}, l_{j}, j=1,2\right\}$. Then for this state the separability condition is give by $\alpha_{k_{1} k_{2}} \alpha_{l_{1} l_{2}}=\alpha_{l_{1} k_{2}} \alpha_{k_{1} l_{2}}$. This equation also gives a general expression for the concurrence

$$
C(|\Psi\rangle)=\left(\mathcal{N} \sum_{l_{1}>k_{1}=0}^{N_{1}-1} \sum_{l_{2}>k_{2}=0}^{N_{2}-1}\left|\alpha_{k_{1} k_{2}} \alpha_{l_{1} l_{2}}-\alpha_{l_{1} k_{2}} \alpha_{k_{1} l_{2}}\right|^{2}\right)^{1 / 2}
$$

of a general bipartite state 3. Now, we will discuss the direct decompositions which is the one interesting property of tensor product. Let $\mathcal{V}_{1}$ and $\mathcal{V}_{2}$ be two complex vector spaces and there is a direct decompositions of these space as $\mathcal{V}_{1}=\sum_{r} \mathcal{V}_{1}^{r}$ and $\mathcal{V}_{2}=\sum_{s} \mathcal{V}_{2}^{s}$. Moreover, assume that the pair $\left(\mathcal{V}_{1} \otimes \mathcal{V}_{2}, \otimes\right)$ is a tensor product of these spaces. Then $\mathcal{V}_{1} \otimes \mathcal{V}_{2}$ is the direct sum of the subspaces $\mathcal{V}_{1}^{r} \otimes \mathcal{V}_{2}^{s}$, that is $\mathcal{V}_{1} \otimes \mathcal{V}_{2}=\sum_{r} \sum_{s} \mathcal{V}_{1}^{r} \otimes \mathcal{V}_{2}^{s}$. The first condition I follows from the observation that $\mathcal{V}_{1} \otimes \mathcal{V}_{2}$ is generated by $\left|\psi_{1}\right\rangle \otimes\left|\psi_{2}\right\rangle$ for $\left|\psi_{j}\right\rangle \in \mathcal{V}_{j}, j=1,2$. But $\left|\psi_{1}\right\rangle \otimes\left|\psi_{2}\right\rangle=\sum_{r} \sum_{s}\left|\psi_{1}^{r}\right\rangle \otimes\left|\psi_{2}^{s}\right\rangle$ for $\left|\psi_{1}\right\rangle=\sum_{r}\left|\psi_{1}^{r}\right\rangle$ and $\left|\psi_{2}\right\rangle=\sum_{s}\left|\psi_{2}^{s}\right\rangle$ with $\left|\psi_{1}^{r}\right\rangle \in \mathcal{V}_{1}^{r}$ and $\left|\psi_{2}^{s}\right\rangle \in \mathcal{V}_{2}^{s}$. Thus $\mathcal{V}_{1} \otimes \mathcal{V}_{2}$ is the sum of the subspaces $\mathcal{V}_{1}^{r} \otimes \mathcal{V}_{2}^{s}$. It is more difficult to show that the decomposition is direct and the proof can be found in [1].

\section{Multilinear mapping and multipartite states}

In this section we will give an introduction to multilinear mapping and tensor product. The relation between the multilinear mapping and tensor product gives the product states of multipartite states. Let $\mathcal{V}_{1}, \mathcal{V}_{2}, \ldots, \mathcal{V}_{m}$ be $m$ complex vector spaces. Then the mapping $\Phi: \mathcal{V}_{1} \times \mathcal{V}_{2} \times \cdots \times \mathcal{V}_{m} \longrightarrow \mathcal{M}$ is called $m$-linear 
if for every $j$ with $1 \leq j \leq m$ we have

$$
\begin{aligned}
& \Psi\left(\left|\psi_{1}\right\rangle, \ldots,\left|\psi_{j-1}\right\rangle, \lambda\left|\psi_{j}\right\rangle+\mu\left|\phi_{j}\right\rangle,\left|\psi_{j+1}\right\rangle, \ldots,\left|\psi_{m}\right\rangle\right. \\
& =\lambda \Psi\left(\left|\psi_{1}\right\rangle, \ldots,\left|\psi_{j-1}\right\rangle,\left|\psi_{j}\right\rangle,\left|\psi_{j+1}\right\rangle, \ldots,\left|\psi_{m}\right\rangle\right. \\
& +\mu \Psi\left(\left|\psi_{1}\right\rangle, \ldots,\left|\psi_{j-1}\right\rangle,\left|\phi_{j}\right\rangle,\left|\psi_{j+1}\right\rangle, \ldots,\left|\psi_{m}\right\rangle,\right.
\end{aligned}
$$

where $\left|\psi_{j}\right\rangle,\left|\phi_{j}\right\rangle \in \mathcal{V}_{j}$ and $\lambda, \mu \in \mathcal{K}$. We also denote the image of the mapping $\Psi$ by $\operatorname{Im} \Psi$, that is, the subspace of $\mathcal{M}$ which is generated by the vectors $\Psi\left(\left|\psi_{1}\right\rangle,\left|\psi_{2}\right\rangle, \ldots,\left|\psi_{m}\right\rangle\right.$. Moreover, let $\mathcal{L}\left(\mathcal{V}_{1}, \mathcal{V}_{2}, \ldots, \mathcal{V}_{m} ; \mathcal{M}\right)$ be the set of all $m$ linear maps $\mathcal{L}\left(\mathcal{V}_{1}, \mathcal{V}_{2}, \ldots, \mathcal{V}_{m} \longrightarrow \mathcal{M}\right)$. Then we can obtain a linear structure in $\mathcal{L}\left(\mathcal{V}_{1}, \mathcal{V}_{2}, \ldots, \mathcal{V}_{m} ; \mathcal{M}\right)$ by defining the following operations

$(\Psi+\Phi)\left(\left|\psi_{1}\right\rangle,\left|\psi_{2}\right\rangle, \ldots,\left|\psi_{m}\right\rangle\right)=\Psi\left(\left|\psi_{1}\right\rangle,\left|\psi_{2}\right\rangle, \ldots,\left|\psi_{m}\right\rangle\right)+\Phi\left(\left|\psi_{1}\right\rangle,\left|\psi_{2}\right\rangle, \ldots,\left|\psi_{m}\right\rangle\right)$

and $(\lambda \Psi)\left(\left|\psi_{1}\right\rangle,\left|\psi_{2}\right\rangle, \ldots,\left|\psi_{m}\right\rangle\right)=\lambda \Psi\left(\left|\psi_{1}\right\rangle,\left|\psi_{2}\right\rangle, \ldots,\left|\psi_{m}\right\rangle\right)$.

Definition 3.0.3 Let $\mathcal{V}_{1}, \mathcal{V}_{2}, \ldots, \mathcal{V}_{m}$ be $m$ complex vector spaces and consider he -linear mapping

$$
\Phi: \mathcal{V}_{1} \times \mathcal{V}_{2} \times \cdots \times \mathcal{V}_{m} \longrightarrow \mathcal{M}
$$

Then the pair $(\Phi, \mathcal{M})$ is called a tensor product if and only if the following important conditions are satisfied:

- $\mathrm{I}_{\otimes}$ : the image of bilinear mapping is equal the target space $\operatorname{Im} \Phi=\mathcal{M}$

- $\mathrm{II}_{\otimes}$ : If there is bilinear mapping $\Psi: \mathcal{V}_{1} \times \mathcal{V}_{2} \times \cdots \times \mathcal{V}_{m} \longrightarrow \mathcal{N}$, where $\mathcal{N}$ is a arbitrary complex vector space, then there exists a linear mapping $\Theta \mathcal{M} \longrightarrow \mathcal{N}$ such that the following diagram

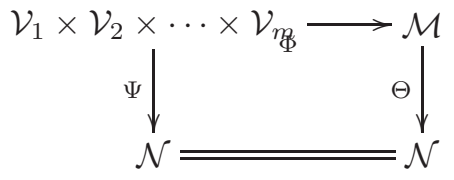

is commutative, that is $\Psi=\Theta \circ \Phi$.

We can denote the tensor product $(\Phi, \mathcal{M})$ of spaces $\mathcal{V}_{j}$ by $\left(\mathcal{V}_{1} \otimes \mathcal{V}_{2} \otimes \cdots \otimes\right.$ $\left.\mathcal{V}_{m}, \otimes^{m}\right)$ and $\Phi\left(\left|\phi_{1}\right\rangle,\left|\phi_{2}\right\rangle, \ldots,\left|\phi_{m}\right\rangle\right)=\left|\phi_{1}\right\rangle \otimes\left|\phi_{2}\right\rangle \otimes \cdots \otimes\left|\phi_{m}\right\rangle$. Now, let $\mathcal{V}_{1}, \mathcal{V}_{2}, \ldots, \mathcal{V}_{m}$ be $m$ complex vector spaces and $\mathcal{V}_{1} \otimes \mathcal{V}_{2} \otimes \cdots \otimes \mathcal{V}_{m}$ be a tensor product for these spaces. Moreover, let $\mathcal{L}\left(\mathcal{V}_{1} \otimes \mathcal{V}_{2} \otimes \cdots \otimes \mathcal{V}_{m} ; \mathcal{M}\right)$ denotes linear mapping $\mathcal{V}_{1} \otimes \mathcal{V}_{2} \otimes \cdots \otimes \mathcal{V}_{m} \longrightarrow \mathcal{M}$ and $\mathcal{L}\left(\mathcal{V}_{1}, \mathcal{V}_{2}, \ldots, \mathcal{V}_{m} ; \mathcal{M}\right)$ denotes multilinear mapping $\mathcal{V}_{1} \times \mathcal{V}_{2} \times \cdots \times \mathcal{V}_{m} \longrightarrow \mathcal{M}$. Then, we have following isomorphism

$$
\mathcal{L}\left(\mathcal{V}_{1} \otimes \mathcal{V}_{2} \otimes \cdots \otimes \mathcal{V}_{m} ; \mathcal{M}\right) \longrightarrow \mathcal{L}\left(\mathcal{V}_{1}, \mathcal{V}_{2}, \ldots, \mathcal{V}_{m} ; \mathcal{M}\right)
$$

which is defined by $\Phi(\Theta)=\Theta \circ \otimes$ for all $\Theta \in \mathcal{L}\left(\mathcal{V}_{1} \otimes \mathcal{V}_{2} ; \mathcal{M}\right)$. The proof follows from conditions $\mathrm{I}_{\otimes}$ and $\mathrm{II}_{\otimes}$ for tensor product. Moreover, the correspondence between the linear map $\Psi \in \mathcal{L}\left(\mathcal{V}_{1} \otimes \mathcal{V}_{2} \otimes \cdots \otimes \mathcal{V}_{m} ; \mathcal{M}\right)$ and $\Theta \in$ $\mathcal{B}\left(\mathcal{V}_{1}, \mathcal{V}_{2}, \ldots, \mathcal{V}_{m} ; \mathcal{M}\right)$ is visualize in following commutative diagram

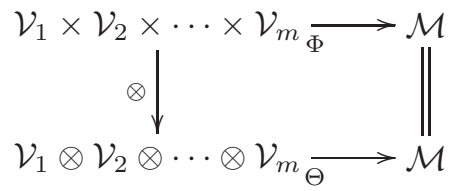

Thus in general case we have the following proposition 
Proposition 3.0.4 Let $\Psi \in \mathcal{L}\left(\mathcal{V}_{1} \otimes \mathcal{V}_{2} \otimes \cdots \otimes \mathcal{V}_{m} ; \mathcal{M}\right)$ be a mulilinear map and $\Theta \in \mathcal{L}\left(\mathcal{V}_{1}, \mathcal{V}_{2}, \ldots, \mathcal{V}_{m} ; \mathcal{M}\right)$ be induced linear map. Then $\Theta$ is surjective and injective if and only if $\Phi$ satisfies the condition $\mathrm{I}_{\otimes}$ and $\mathrm{II}_{\otimes}$ of tensor respectively.

Let $\mathcal{V}_{1}, \mathcal{V}_{2}, \ldots, \mathcal{V}_{m}$ be complex vector spaces. Then the mapping

$$
\Psi: \mathcal{L}\left(\mathcal{V}_{1}\right) \times \mathcal{L}\left(\mathcal{V}_{2}\right) \times \cdots \times \mathcal{L}\left(\mathcal{V}_{m}\right) \longrightarrow \mathcal{L}\left(\mathcal{V}_{1} \otimes \mathcal{V}_{2} \otimes \cdots \otimes \mathcal{V}_{m}\right)
$$

given by $\Psi\left(\Theta_{1}, \ldots, \Theta_{m}\right)\left(\left|\psi_{1}\right\rangle \otimes \cdots \otimes\left|\psi_{m}\right\rangle\right)=\Theta_{1}\left(\left|\psi_{1}\right\rangle\right) \cdots \Theta_{m}\left(\left|\psi_{m}\right\rangle\right)$ is a tensor product for the space $\mathcal{L}\left(\mathcal{V}_{j}\right)$.

We will give an example to visualize the relation between our $m$-linear construction and multipartite product states. Let us consider a multipartite states where $\mathcal{V}_{j}=\mathbb{C}^{N_{j}}$ for all $1 \leq j \leq m$ and a general state is given by

$$
|\Psi\rangle=\sum_{i_{1}, i_{2}, \ldots, i_{m}=0}^{N_{1}-1, N_{2}-1, \ldots, N_{m}-1} \alpha_{i_{1} i_{2} \cdots i_{m}}\left|i_{1}\right\rangle \otimes\left|i_{2}\right\rangle \otimes \cdots\left|i_{m}\right\rangle .
$$

Then we have the following commutative diagram

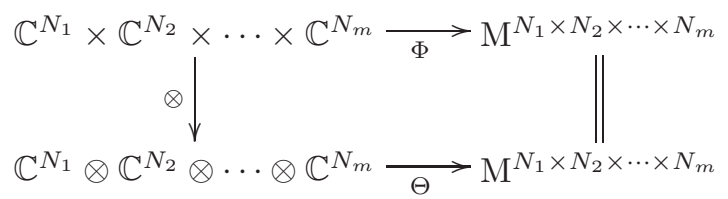

where $\mathrm{M}^{N_{1} \times N_{2} \times \cdots \times N_{m}}=\left(\alpha_{i_{1} i_{2} \cdots i_{m}}\right)_{1 \leq i_{j} \leq N_{j}}$, is a multi-box matrix which is defined as follows

$$
\mathrm{M}^{N_{1} \times \cdots \times N_{m}}=\left\{\alpha_{i_{1} \ldots i_{m}} \in \mathbb{C}^{N_{1}} \times \cdots \times \mathbb{C}^{N_{m}}: \mathcal{S}_{1 \leq j \leq m}^{k_{j} l_{j}}=0, \forall, j=1,2, \ldots, m\right\},
$$

where

$$
\begin{aligned}
\mathcal{S}_{1 \leq j \leq m}^{k_{j} l_{j}}= & \alpha_{k_{1} k_{2} \cdots k_{m}} \alpha_{l_{1} l_{2} \cdots l_{m}}- \\
& \alpha_{k_{1} k_{2} \cdots k_{j-1} l_{j} k_{j+1} \cdots k_{m}} \alpha_{l_{1} l_{2} \cdots l_{j-1} k_{j} l_{j+1} \cdots l_{m}}
\end{aligned}
$$

This construction also gives a general expression for the concurrence

$$
C(|\Psi\rangle)=\left(\mathcal{N} \sum_{l_{1}>k_{1}=0}^{N_{1}-1} \cdots \sum_{l_{3}>k_{3}=0}^{N_{3}-1}\left|\mathcal{S}_{1 \leq j \leq 3}^{k_{j} l_{j}}\right|^{2}\right)^{1 / 2}
$$

of a general three-partite state. Note also that for a mixed state a measure of entanglement can be constructed by taking the infimum over all pure decomposition of a given state using above expression for concurrence. This is our main result for general multipartite states. This result is also related to the construction of Segre variety given in [3]. We can also construct a measure of entanglement for general multipartite states based on the multidimensional matrix $\mathrm{M}^{N_{1} \times N_{2} \times \cdots \times N_{m}}$ with some additional structures 4 .

Acknowledgments: The author acknowledges the financial support of the Japan Society for the Promotion of Science (JSPS). 


\section{References}

[1] W.H. Greub, Multiliear algebra, Springer-Verlag, Berlin, 1967.

[2] W. K. Wootters, Phys. Rev. Lett. 80, 2245 (1998).

[3] H. Heydari and G. Björk, J. Phys. A: Math. Gen. 38 (2005) 3203-3211.

[4] H. Heydari, J. Phys. A: Math. Gen. 39 (2006) 9839-9844. 